\title{
Über die elektrischen Eigenschaften von InSb
}

\author{
Von H. Weiss \\ Aus dem Forschungslaboratorium der Siemens-Schuckertwerke in Erlangen \\ (Z. Naturforschg. 8a, 463-469 [1953]; eingegangen am 24. Juni 1953)
}

In Ergänzung früherer, von H. W elker veröffentlichter Angaben werden Ergebnisse der Messungen von Leitfähigkeit, Hall-Effekt und magnetischer Widerstandsänderung an InSb mitgeteilt. Mit Hilfe des Hall-Effektes wurden je nach Störstellengehalt Präparate sowohl mit n- als auch mit p-Leitung gefunden. Bei Zimmertemperatur erhält man als größte Elektronenbeweglichkeit $41000 \mathrm{~cm}^{2} /$ Voltsec, während die höchste Widerstandsänderung in einem Magnetfeld von 10000 Gauß $150 \%$ beträgt.

$\mathrm{N}$ ach Überlegungen von Welker ${ }^{1}$ sind Verbindungen der Elemente der 3. und 5. Gruppe des periodischen Systems in ihren physikalischen Eigenschaften den Elementen der 4. Gruppe sehr ähnlich. So besitzt InSb Zinkblendenstruktur mit derselben Gitterkonstante wie das im periodischen System zwischen Indium und Antimon stehende graue Zinn. In der vorliegenden Arbeit werden in Ergänzung früherer Angaben 1, 2 die Ergebnisse der Messungen von Leitfähigeit, Hall-Effekt und magnetischer Widerstandsänderung an In Sb mitgeteilt.

\section{A. Herstellung der Präparate}

Sechs verschiedene Präparate von InSb wurden dadurch erhalten, daß man die Komponenten In und $\mathrm{Sb}$ vor dem stöchiometrischen Zusammenschmelzen einer chemischen Reinigung unterzog, die für die einzelnen Proben bei jeweils verschiedenen Reinheitsgraden abgebrochen wurde. Nach dem Zusammenschmelzen wurden aus dem Regulus Stäbe von $5 \times 5 \mathrm{~mm}^{2}$ Querschnitt herausgeschnitten und bezüglich des Ganges der spezifischen Leitfähigkeit mit der Länge untersucht. Dazu wurde ein Strom durch den Stab geschickt und mit Hilfe von zwei Sonden, deren Abstand durch einen Mikrometerschlitten meßbar verändert werden konnte, der Spannungsabfall längs des Stabes gemessen. Zur weiteren. Untersuchung wurden nur Proben mit sehr geringer Abweichung von der Homogenität mit einer Länge von etwa $35 \mathrm{~mm}$ verwendet. Die Nummerierung der Präparate ist so gewählt, daß Nr. 1 die ungereinigte Ausgangssubstanz darstellt (ca. 0,5\% Verunreinigungen) und mit einer größeren Nummer ein höherer Reinheitsgrad verknüpft ist. Die Verunreinigungen der Probe 6 bestehen nach spektroskopischen Untersuchungen im wesentlichen aus Ca und Mg. Die Proben sind polykristallin mit Ausnahme von Nr. 4. Dieses Präparat ist ein aus der Schmelze gezogener

${ }^{1}$ H. Welker, Z. Naturforschg. 7 a, 744 [1952].

${ }^{2}$ H. W elker, Z. Naturforschg. 8a, 248 [1953].

${ }^{3}$ R. Gremmelmaier u. O. Madelung, Z. Naturforschg. $8 \mathbf{a}, 333$ [1953].
Einkristall ${ }^{3}$. Er ist so orientiert, daß zwei gegenüberliegende Prismenflächen bis auf eine Abweichung von 7 Grad mit einer Rhombendodekaederfläche (110) zusammenfallen. Die Stabachse hat die Richtung $\left[2_{1}^{-3}\right]^{4}$.

\section{B. Meßmethode}

Die Messungen von Leitfähigkeit, transversaler magnetischer Widerstandsänderung und Hall-Effekt erfolgten durch Kompensation nach der Gleichstrommethode in derselben Apparatur (Abb. 1). B war eine den Probenstrom $(1-500 \mathrm{~mA})$ liefernde Spannungsquelle von 12 Volt, $R$ ein Widerstand zum Einstellen des Stromes. Die Drähte für die Stromzuführungen wurden entweder durch Federn oder durch Anlöten mit den Enden der Probe verbunden. Die Sonden $\mathbf{S}_{1}$ und $\mathbf{S}_{2}$ an der Vorderseite der Probe und die Sonde $\mathbf{S}_{3}$ an der Rückseite in gleicher Höhe mit $\mathbf{S}_{2}$ bestanden aus Messingspitzen, die durch Federn angedrückt wurden.

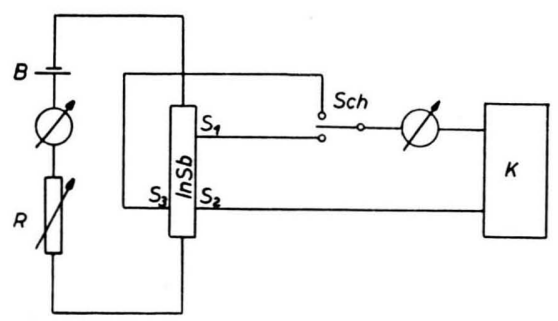

Abb. 1. Schaltskizze zu den Messungen.

Der Abstand der Sonden voneinander konnte entsprechend der Größe des Präparates verändert werden. Mit Hilfe eines thermokraftfreien Kompensators K nach Diesselhorst wurde je nach der Stellung des Schalters Sch entweder die Spannung zwischen $S_{1}$ und $S_{2}$ oder die Hall-Spannung zwischen $\mathbf{S}_{2}$ und $\mathbf{S}_{3}$ gemessen.

Die Probe mit Stromzuführungen und Sonden konnte im Bereiche von $-210^{\circ} \mathrm{C}$ bis $+300^{\circ} \mathrm{C}$ in ein

4 Die Herstellung der InSb-Präparate mit extrem hoher magnetischer Widerstandsänderung sowie die dazu notwendigen metallurgischen Untersuchungen von Herrn O. G. Folberth durchgeführt. 
Magnetfeld von 7500 Gauß gebracht werden. Aus den Spannungen zwischen den $S_{1}$ und $S_{2}$, gemessen ohne und mit Magnetfeld bei festgehaltenem Probenstrom, ließen sich spezifische Leitfähigkeit und transversale Widerstandsänderung berechnen. Durch Variieren des Stromes durch die Probe über eine Größenordnung wurde bei jeder Temperatur, bei der die Leitfähigkeit untersucht wurde, das Ohmsche Gesetz nachgeprüft. Die Beziehung Strom-Spannung war stets linear.

Die Hall-Spannung wurde jeweils für die beiden Richtungen von Probenstrom und magnetischem Feld, außerdem bei 7500 und bei 2500 Gauß gemessen, um Abweichungen von der Linearität zwischen Hall-Spannung und magnetischer Feldstärke festzustellen. Die Proportionalität zwischen Hall-Spannung und Probenstrom wurde wiederholt nachgeprüft. Da sich bei den Messungen zwischen $100^{\circ} \mathrm{C}$ und $300^{\circ} \mathrm{C}$ die Probe in einem umlaufenden Ölbad befand, konnte der Ettingshausen-Effekt die Hall-Spannung nur bei Temperaturen unterhalb $100^{\circ} \mathrm{C}$ verfälschen. Bei den Messungen an InSb war durch diesen Effekt jedoch keine Beeinträchtigung der Meßgenauigkeit zu befürchten. Infolge der hohen Beweglichkeiten liegen die Hall-Spannungen in der Größenordnung einiger Millivolt, zudem sinkt die differentielle Thermokraft mit abnehmender Temperatur. Bei Zimmertemperatur wurde die HallSpannung vergleichsweise mit der Wechselstrommethode gemessen. Im Bereich von $250-5000 \mathrm{~Hz}$ ergab sich keine Abweichung von den Gleichstromwerten, die größer war, als der Meßgenauigkeit von $3 \%$ entsprach.

\section{Meßapparatur}

Die Anlage für die Messungen zwischen $-210^{\circ} \mathrm{C}$ und $100^{\circ} \mathrm{C}$ ist in einem Schnitt senkrecht zu den magnetischen Feldlinien in Abb. 2 a dargestellt. In einem evakuierbaren zylindrischen Messinggefäß mit dem Schliff $P$ für den Anschluß der Diffusionspumpe befinden sich zwei Behälter aus Kupfer, ein innerer zylindrischer, $B_{1}$, und ein diesen umschließender zweiter ringförmiger, $B_{2}$. Sie können von außen durch die zugleich als Halterung dienenden Rohre aus Kontracid bzw. Neusilber mit flüssigem Sauerstoff gefüllt werden. Die Temperatur des inneren Topfes wird durch Abpumpen bis auf etwa $-210^{\circ} \mathrm{C}$ erniedrigt oder durch Einbringen einer Heizspirale auf $100^{\circ} \mathrm{C}$ erhöht. Dazwischen liegende Temperaturen lassen sich konstant über mehrere Minuten herstellen, da die Wärmekapazität des inneren Topfes groß gegenüber der sehr geringen Wärmeableitung des Kontracidrohres ist. Die zu untersuchende Probe ist mit dem einen Ende, zusammen mit einem Manganin-Konstantan-Thermoelement, an den Kupferblock $\mathrm{Cu}$ angelötet. Sonden und Stromzuführung sind angedeutet. $W_{1}$ und $W_{2}$ sind zwei $0,5 \mathrm{~mm}$ dicke, außen versilberte Kupferbleche zum Schutz gegen Wärmezustrahlung. Das Erreichen des Temperaturgleichgewichtes ließ sich leicht feststellen. In diesem Falle verschwand nämlich die Thermospannung zwischen den Sonden.

Der obere Teil der Meßapparatur besitzt Rotationssymmetrie. Der untere mit der Probenhalterung ist in der Richtung parallel zu den magnetischen Feldlinien auf $12 \mathrm{~mm}$ Ausdehnung beschränkt (Abb. $2 \mathrm{~b}$ ), um ein auf die Länge der Probe homogenes Magnetfeld von 7500 Gauß zu ermöglichen. Die Polschuhe des Elektromagneten werden durch Einsätze im Vakuumgefä $B$ bis auf einen Spalt von $15 \mathrm{~mm}$ fortgesetzt.

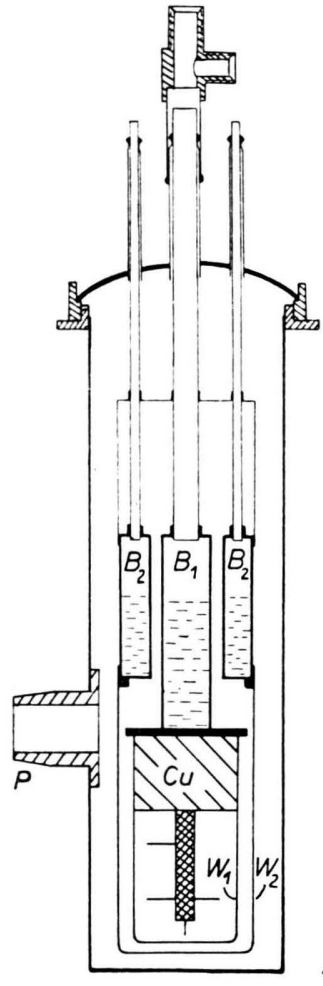

a)

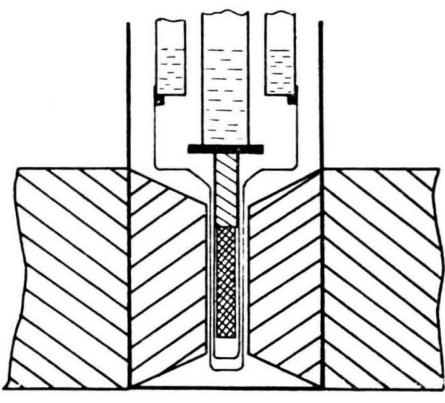

b)
Abb. 2. Schnitt durch die Meßapparatur für Temperaturen unterhalb $100^{\circ} \mathrm{C}$, a) senkrecht zu den magnetischen Feldlinien; b) parallel zu den magnetischen Feldlinien.

Für die Hall-Messungen zwischen $100^{\circ} \mathrm{C}$ und $300^{\circ} \mathrm{C}$ befand sich die Probe mit den Sonden in einem schmalen Gehäuse, das in einen Magnetspalt von $10 \mathrm{~mm}$ gebracht werden konnte. Der Behälter wurde während der Messung mit Hilfe eines Umlaufthermostaten von heißem Öl durchströmt. Zwischen $300^{\circ} \mathrm{C}$ und $450^{\circ} \mathrm{C}$ wurde nur die Leitfähigkeit in einem elektrischen Ofen gemessen.

Zur Herstellung des Magnetfeldes diente ein Elektromagnet, der bei einem Strom von 8 Amp. in einem Spalt von $15 \mathrm{~mm}$ Abstand und $36 \mathrm{~cm}^{2}$ Fläche ein homogenes Feld von 7500 Gauß lieferte. Die Messung des Feldes erfolgte mit einer geeichten Wismutspirale von Hartmann und Braun.

\section{Meßergebnisse}

a) Spezifische Leitfähigkeit

Zunächst wurde die spezifische Leitfähigkeit der 6 Proben InSb im Bereiche von $-210^{\circ} \mathrm{C}$ bis $450^{\circ} \mathrm{C}$ 
untersucht. Die Ergebnisse der Messungen sind in Abb. 3 dargestellt. Die Buchstaben $p, n$ geben an, ob die Ladungsträger im Bereiche der Störleitung Elektronen (n) oder Löcher (p) sind. Man erhält ein Diagramm ähnlich dem, das man von den halbleitenden Elementen der vierten Gruppe des periodischen Systems bereits kennt: die bei tiefen Temperaturen sich um Größenordnungen unterscheidenden, nur wenig von der Temperatur abhängigen spezifischen Leitfähigkeiten der einzelnen Präparate (Nr. 1 bis 6) münden bei hohen Temperaturen schließlich in die Eigenleitungsgerade ein, die einen sehr steilen Anstieg der spez. Leitfähigkeit $\sigma$ mit zunehmender Temperatur aufweist. Die Proben 2 und 6 besitzen nun eine solche Gerade zwischen $100^{\circ}$ und $300^{\circ} \mathrm{C}$ bzw. zwischen $20^{\circ}$ und $200^{\circ} \mathrm{C}$. In diesen Intervallen lassen sich die Leitfähigkeiten durch folgende Gleichung ausdrücken :

$$
\sigma=A \cdot e^{\frac{-\Delta E}{2 k T}},
$$

wo $k=$ Boltzmann-Konstante, $T=$ absolute Temperatur, $\Delta E=$ Breite der verbotenen Zone.

Für $\Delta E$ erhält man aus den Messungen für beide Geraden etwa $0,5 \mathrm{eV}$. Der Faktor $A$ in Gl. (1) ist infolge der parallelen Verschiebung der beiden Geraden für Präp. 6 viermal größer als für Präp. 2.

Oberhalb $300^{\circ} \mathrm{C}$ für Präp. 2 und $200^{\circ} \mathrm{C}$ für Präp. 6 nimmt die spezifische Leitfähigkeit in geringerem Maße zu als sich aus Gl. (1) ergibt. In das letzte gekrümmte Stück der Kurve 6 münden schließlich auch die $\sigma$-Kurven der Präparate 3,4 und 5 ein.

Bemerkenswert ist die Temperaturabhängigkeit von $\sigma$ für die die meisten Störstellen enthaltende Probe 1. Sie besitzt zwischen $-183^{\circ}$ und $450^{\circ} \mathrm{C}$ durchweg einen metallischen Temperaturkoeffizienten der spezifischen Leitfähigkeit.

\section{b) Hall-Konstante}

Um weiteren Aufschluß über den Leitungsmechanismus in InSb zu erhalten, wurde die Hall-Spannung an den verschiedenen Präparaten gemessen und daraus die Hall-Konstante $R$ nach folgender Beziehung errechnet:

$$
R=\frac{V_{\mathrm{H}} d}{i B},
$$

wo $B=$ magnetische Induktion [Voltsec $/ \mathrm{cm}^{2}$ ] (gemessen wurde $B$ in Gauß: $10^{8} \mathrm{Gau} \beta=1$ Voltsec $/$ $\left.\mathrm{cm}^{2}\right), d=$ Dicke der Probe [cm], $i=$ Probenstrom [Amp.], $R=$ Hall-Konstante [ $\mathrm{cm}^{3} /$ Ampsec], $V_{\mathrm{H}}=$

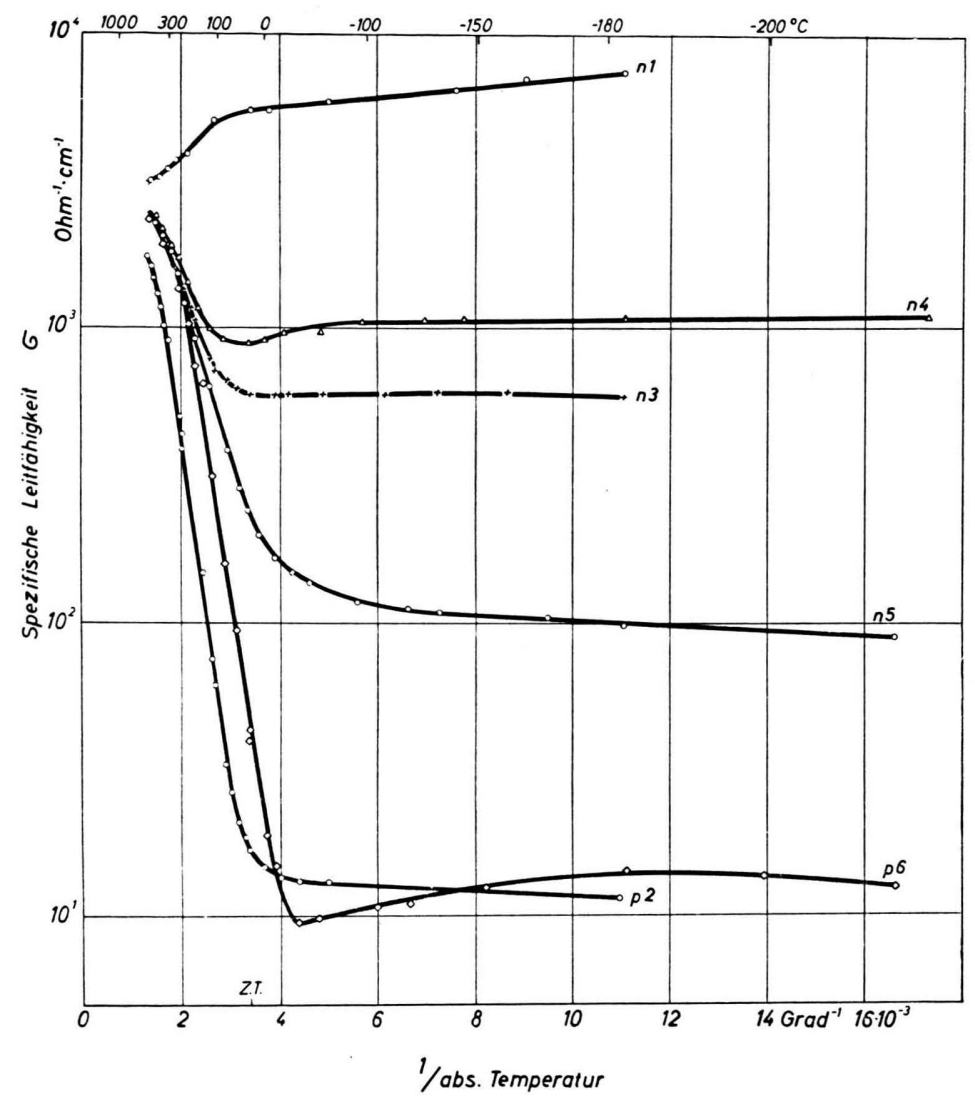

Abb. 3. Spezifische Leitfähigkeit $\sigma$ von 6 InSb-Proben als Funktion der reziproken absoluten Temperatur.

Hall-Spannung [Volt]. Dabei ist $R$ negativ für Elektronen- und positiv für Löcher-Leitung.

Im allgemeinen ist die Hall-Spannung proportional zum Strom $i$ und zur magnetischen Induktion $B$, somit $R$ unabhängig von $i$ und $B$. In dem Temperaturbereich jedoch, in dem infolge des Überganges von der p-Leitung in die Eigenleitung das Vorzeichen der Hall-Konstante von plus nach minus wechselt, ist die Hall-Spannung der magnetischen Feldstärke nicht mehr proportional. Es ist dadurch möglich experimentell zu entscheiden, ob sich die Probe bei einer gegebenen Temperatur noch im Übergangs- 


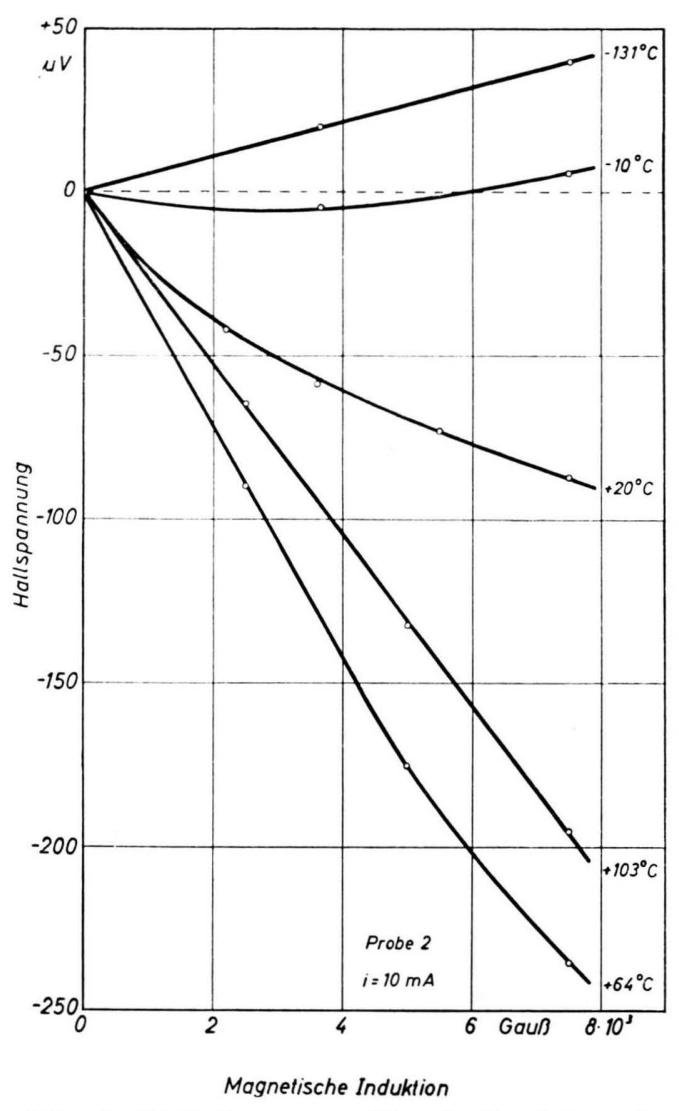

Abb. 4. Hall-Spannung $V_{\mathrm{H}}$ als Funktion der magnetischen Induktion bei verschiedenen Temperaturen, Messungen an Probe $2 ; i=10 \mathrm{~mA}$.

Abb. 5a. Hall-Konstante $R$ in Abhängigkeit von der reziproken absoluten Temperatur für 4 n-leitende Präparate. Hall-Konstante stets negativ.

Abb. 5b. Hall-Konstante $R$ in Abhängigkeit von der reziproken absoluten Temperatur für 2 p-leitende Präparate. Hall-Konstante bei tiefen Temperaturen positiv.

gebiet befindet. Als Beispiel ist in Abb. 4 $V_{\mathrm{H}}$ als Funktion der magnetischen Induktion für verschiedene Temperaturen, gemessen an Probe 2, aufgetragen. Die Kurven sind in der Umgebung von $0^{0} \mathrm{C}$ gekrümmt, bei $-10^{0} \mathrm{C}$ liegt bei 6000 Gauß ein Nulldurchgang der Hall-Spannung. Bei $+103^{0} \mathrm{C}$, d. h. in der Eigenleitung, und bei $-131^{\circ} \mathrm{C}$, in der reinen Störleitung, ist $V_{\mathrm{H}}$ bis $7500 \mathrm{Gau}$ wieder proportional der magnetischen Induktion.

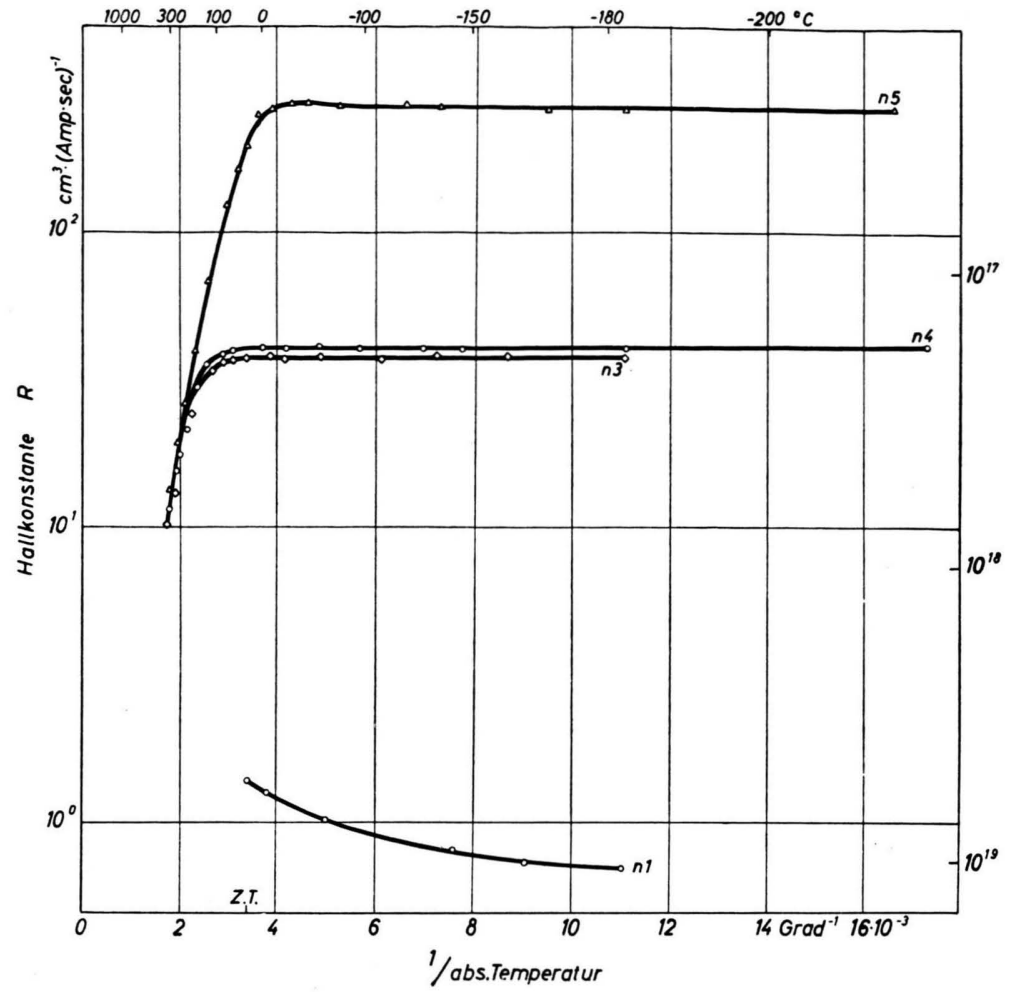

Abb. 5 a.

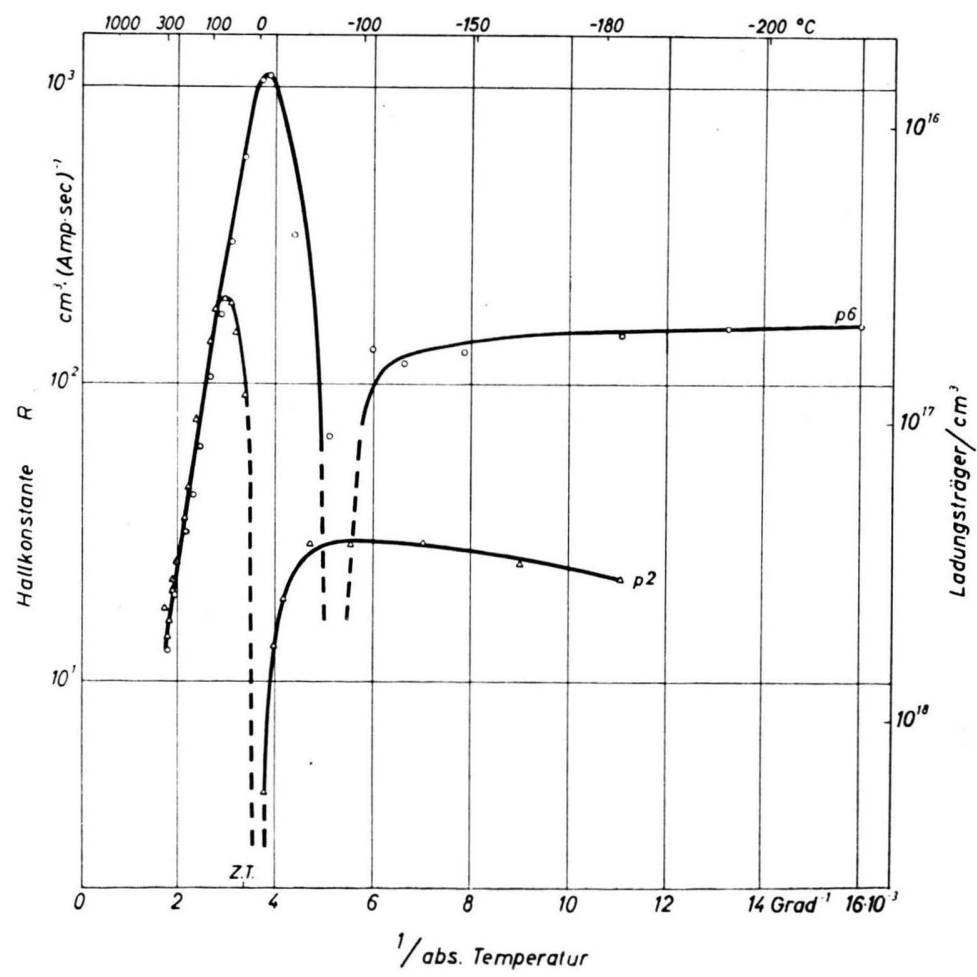

Abb. 5 b. 
Dunlap ${ }^{5}$ hat auf ähnliche Beobachtungen bei Germanium hingewiesen, Madelung und Wel$\mathrm{ker}^{6}$ stellten allgemeine Formeln für die Hall-Spannung bei gemischter Leitung auf und werteten sie für Ge zahlenmäßig aus.

In den Abb. $5 \mathrm{a}$ und $5 \mathrm{~b}$ ist $\log R$, berechnet nach Gl. (2) aus den Meßergebnissen bei 2500 Gauß, in Abhängigkeit von $1 / T$ aufgetragen. Das Vorzeichen von $R$ ist bei den Proben 1, 3, 4, 5 stets negativ, bei 2 und 6 bei tiefen Temperaturen positiv und nach einem Nulldurchgang bei höheren Temperaturen schließlich ebenfalls negativ.

Wesentlich erscheint der Befund, daß alle Präparate, auch wenn die jeweilige Leitfähigkeit nicht durch eine gemeinsame Eigenleitungskurve dargestellt wird, bei höheren Temperaturen die gleiche Hall-Konstante besitzen (z. B. 2 und 6 oberhalb $\left.100^{\circ} \mathrm{C}\right)$.

c) Transversale magnetische Widerstandsänderung

InSb zeigt eine sehr große magnetische Widerstandsänderung $\Delta \varrho / \varrho_{\mathrm{H}}$. ( $\varrho_{\mathrm{H}}$ ist der spezifische Widerstand im Magnetfeld.) In Abb. 6 ist $\Delta \varrho / \varrho_{\mathrm{H}}$, gemessen an den Proben 5 und 6, in Abhängigkeit vom Quadrat der magnetischen Induktion bei Zimmertemperatur aufgetragen. Abb. 7 zeigt $\Delta \varrho / \varrho_{\mathrm{H}}$ als Funktion von $1 / T$. Die beiden Proben geben oberhalb von $200^{\circ} \mathrm{C}$ dieselben Werte, während die Ergebnisse bei tiefen Temperaturen sich um eine Größenordnung unterscheiden.

\section{E. Diskussion der Meßergebnisse}

Aus Leitfähigkeit $\sigma$ und Hall-Konstante $R$ lassen sich die Konzentration der Elektronen $(n)$ und diejenige der Löcher $(p)$, soweit sie am Ladungstransport beteiligt sind, sowie deren Beweglichkeiten $\mu_{\mathrm{n}}$ und $\mu_{\mathrm{p}}$ bestimmen.

\section{a) Konzentration der Ladungsträger}

Betrachten wir zunächst das Störleitungsgebiet. Nach den Gleichungen

$$
n=-\frac{3 \pi}{8 e R}, \quad p=\frac{3 \pi}{8 e R},
$$

wo $e=$ Ladung des Elektrons $\left(1,6 \cdot 10^{-19}\right.$ Ampsec $)$, kann man die Konzentrationen der jeweiligen Ladungsträger berechnen. In den Abb. 5a und $5 \mathrm{~b}$ ist

5 W. C. Dunlap, Physic. Rev. 82, 329 [1951].

${ }^{6}$ O. Madelung u. H. Welker, Z. angew. Physik 5, 12 [1953]. an der rechten Ordinate $\log n$ bzw. $\log p$ nach Gl. (3) aufgetragen. Die Konzentrationen bei der Temperatur der flüssigen Luft geben Aufschluß über den Gang der chemischen Reinigung: In dem Ausgangsmaterial, aus dem Probe 1 hergestellt wurde, sind sowohl Donatoren- als auch Acceptorenstörstellen enthalten. Aus Abb. 5a ist zu ersehen, daß etwa $10^{19}$ Elektronen in $1 \mathrm{~cm}^{3}$ als Ladungsträger wirksam sind. Bei der zunächst durchgeführten Reinigung wurden solche Störstellen aus dem InSb weggenommen, die als Donatoren in das Gitter eingebaut werden. Daher wurde in Präp. 2 ein Überschuß von $2 \cdot 10^{17}$ Löchern $/ \mathrm{cm}^{3}$ gefunden. (Abb. 5 b) Im weiteren Verlaufe wurden vorwiegend Verunreinigungen entfernt die p-Leitung verursachen. Man erhielt dann die nleitenden Proben 3, 4 und 5. Zuletzt gelangte man zu Präp. 6 mit einem Überschuß von $5 \cdot 10^{16}$ Acceptoren.

Aus dem nahezu konstanten Verlauf der HallKonstante bei tiefen Temperaturen muß man auf eine vollständige Ionisierung der Donatoren und Acceptoren und damit auf eine sehr kleine Abtrennarbeit schließen.

Im Bereiche der Eigenleitung $\left(n=p=n_{i}\right) \mathrm{mu} \beta$ man an Stelle von Gl. (3) folgende Beziehung verwenden:

$$
R=\frac{-3 \pi}{8 e n_{i}} \cdot \frac{b-1}{b+1}, \text { wo } b=\mu_{\mathbf{n}} / \mu_{\mathbf{p}} .
$$

Nimmt man b, das Verhältnis von Elektronen- zu Löcherbeweglichkeit, temperaturunabhängig an, so liefert die Hall-Konstante $R$ auch in der Eigenleitung den Zusammenhang zwischen der Konzentration der Elektronen und der Temperatur T. Nach Fröhlich ${ }^{7}$ hängt die Trägerkonzentration in der Eigenleitung in folgender Weise von $T$ ab:

$$
n_{i}=2\left(\frac{2 \pi \sqrt{m_{\mathrm{n}} m_{\mathrm{p}}} k T}{h^{2}}\right)^{3 / 2} \cdot e^{-\frac{\Delta E^{\prime}}{2 k T}}
$$

wo $k$ Boltzmann-Konstante, $T$ absolute Temperatur, $h$ Plancksches Wirkungsquantum, $m_{\mathrm{n}}$ scheinbare Elektronenmasse, $m_{\mathrm{p}}$ scheinbare Löchermasse.

Aus (4) und (5) ergibt sich:

$$
R \sim \frac{1}{n_{i}} \sim T^{-3 / 2} \cdot e^{-\frac{\Delta E^{\prime}}{2 k T}} .
$$

Wendet man (6) auf den allen Präparaten gemeinsamen Abfall der Hall-Konstante bei hohen Temperaturen an, so erhält man für $\Delta E^{\prime}$ etwa $0,4 \mathrm{eV}$.

7 H. Fröhlich, Elektronentheorie der Metalle, Springer-Verlag Berlin 1936. 

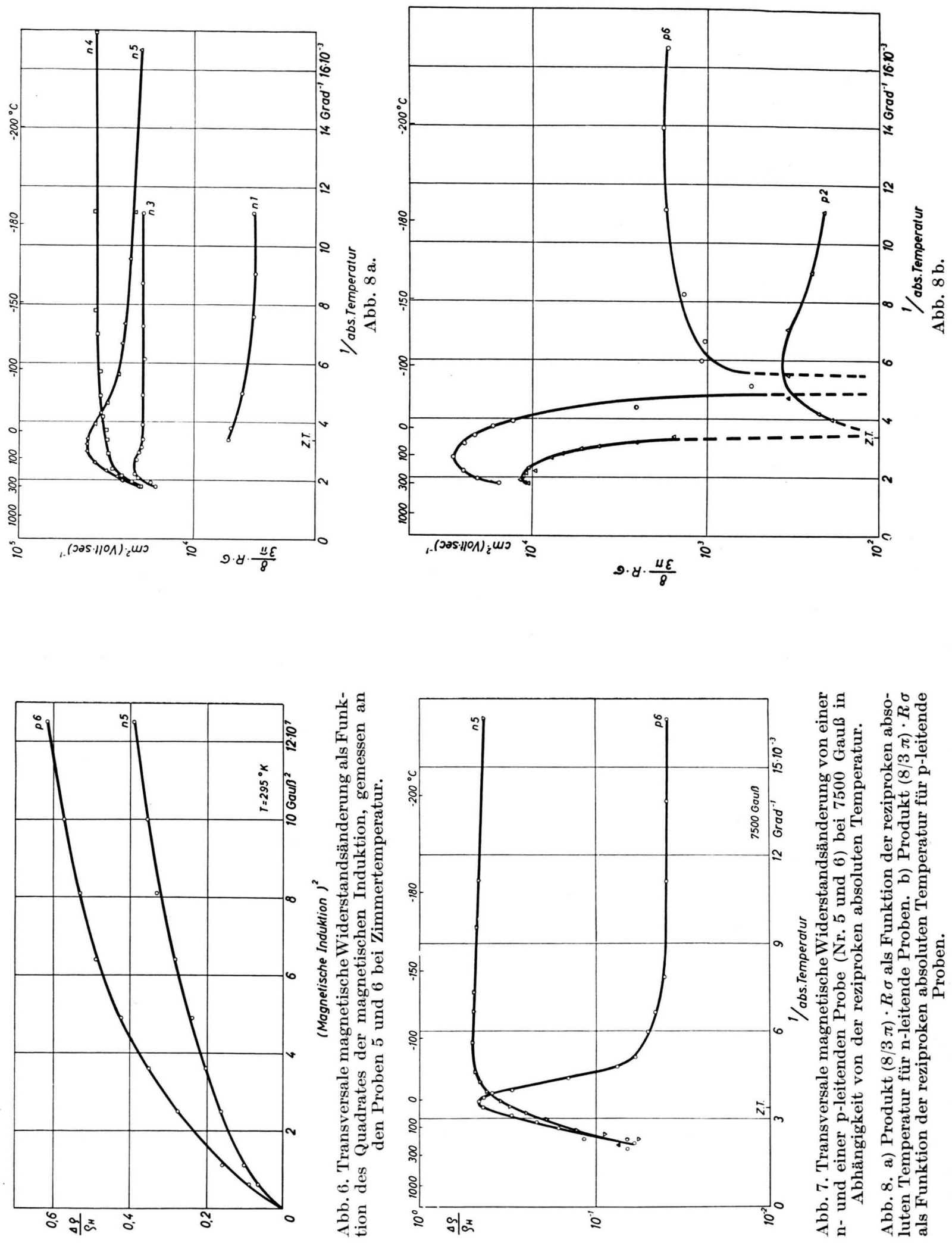


\section{b) Beweglichkeit der Ladungsträger}

Die Beweglichkeit der Ladungsträger wird in der Störleitung durch das Produkt $8 R \sigma / 3 \pi$ angegeben. Diese Größe ist für die 6 untersuchten Proben aus den Meßwerten für $R$ und $\sigma$ berechnet und in den Abb. 8a und 8b in Abhängigkeit von $1 / T$ dargestellt. Die höchste Elektronenbeweglichkeit wurde an Probe 5 bei Zimmertemperatur mit $41000 \mathrm{~cm}^{2} /$ Voltsec, die größte Löcherbeweglichkeit an Probe 6 bei $-200^{\circ} \mathrm{C}$ mit $1800 \mathrm{~cm}^{2} /$ Voltsec gemessen. Aus Abb. $8 \mathrm{a}$ und $8 \mathrm{~b}$ geht hervor, daß die Werte für $\mu_{\mathbf{n}}$ und $\mu_{\mathrm{p}}$ durch die Reinigung des InSb um eine GröBenordnung höher werden. Dieser Befund stimmt qualitativ mit der Formel von Con well und Weiß $\mathrm{kopf}^{8}$ überein. Diese besagt, daß die Beweglichkeit bei Streuung an ionisierten Störstellen der Zahl der Streuzentren umgekehrt proportional ist. Ein quantitativer Vergleich der Meßergebnisse von Trägerkonzentration und Beweglichkeit mit dieser Formel ist jedoch nicht möglich, da die Konzentration von ionisierten Störstellen infolge gleichzeitiger Anwesenheit von Acceptoren und Donatoren bei den untersuchten Präparaten größer als die durch HallEffekt festgestellte Konzentration der Ladungsträger sein kann.

In der Eigenleitung ist das Produkt $8 R \sigma / 3 \pi$ gleich der Differenz von Elektronen- und Löcherbeweglichkeit. Da nun $\mu_{\mathrm{n}}$ in der Störleitung 10 bis 20 mal größer als $\mu_{\mathrm{p}}$ und damit $b>10$ ist, neigt man dazu anzunehmen, daß $8 R \sigma / 3 \pi$ in der Eigenleitung etwa gleich der Elektronenbeweglichkeit $\mu_{\mathrm{n}}$ ist.

Die Abb. $8 \mathrm{a}$ und $8 \mathrm{~b}$ zeigen bei Temperaturen unterhalb $0^{0} \mathrm{C}$ nur eine geringe Abhängigkeit der Beweglichkeiten von $T$. Oberhalb $100^{\circ} \mathrm{C}$ fällt $\mu_{\mathrm{n}}$ jedoch mit wachsendem $T$ ab und zwar stärker als die Beziehung $\mu \sim T^{-3 / 2}$ für Streuung, durch thermische Gitterschwingungen angibt. Dies erklärt den Befund, daß die Leitfähigkeit bei $300^{\circ} \mathrm{C}$ keine exponentielle Abhängigkeit von $T$ mehr besitzt.

Die Eigenleitungsgeraden der Proben 2 und 6 sind bei gleicher Hall-Konstante um den Faktor 4

${ }^{8}$ E. Conwell u. V. F. Weißkopf, Physic. Rev. 77, 388 [1950]. verschoben (vgl. Abb. 3 und 5 b). Ursache hierfür ist die größere Reinheit der Probe 6 . Diese besitzt eine größere Beweglichkeit als 2.

Mit der Annahme $b>10$ erhält man aus der HallKonstante eine Aussage über die Konzentration der Elektronen in der Eigenleitung. Aus Gl. (4) folgt nämlich :

$$
n_{i} \approx \frac{-3 \pi}{8 e R}
$$

Aus den Messungen von $R$ erhält man für $n_{i}$ etwa $10^{16}$ Elektronen und Löcher $/ \mathrm{cm}^{3}$ bei Zimmertemperatur (Abb. 5 b). $\mathrm{Zu}$ demselben Ergebnis gelangt man, wenn man in Gl. (5) für $\sqrt{m_{\mathrm{n}} m_{\mathrm{p}}}$ die Masse des freien Elektrons und für $\Delta E^{\prime} 0,4 \mathrm{eV}$ einsetzt.

Die hohe, bisher bei keinem anderen Körper gefundene Elektronenbeweglichkeit bedingt eine große Widerstandsänderung im transversalen Magnetfeld. Nach Fröhlich ${ }^{7}$ besteht in schwachen Feldern die Beziehung:

$$
\Delta \varrho / \varrho_{\mathrm{H}}=0,38 \mu^{2} B^{2} .
$$

Aus den Anfangssteigungen der beiden Kurven in Abb. 6 erhält man nach Gl. (6) folgende Beweglichkeiten: Probe $518000 \mathrm{~cm}^{2} /$ Voltsec und Probe 6 $21000 \mathrm{~cm}^{2} /$ Voltsec. Die entsprechenden Werte aus dem Hall-Effekt sind 41000 und $20000 \mathrm{~cm}^{2} /$ Voltsec. Letzterer Wert ist möglicherweise zu tief gemessen, da sich Probe 6 bei Zimmertemperatur noch im Übergangsgebiet zwischen Stör- und Eigenleitung befindet und das Produkt $8 R \sigma / 3 \pi$ mit zunehmender Temperatur noch ansteigt (Abb.6 b). Die überraschend hohen Werte der magnetischen Widerstandsänderung - wie sie z. B. bei Probe 6 vorliegen wurden inzwischen auch von Pearson und Tanen baum ${ }^{9}$ an hochgereinigtem InSb gefunden.

Herrn Dr. W elker danke ich für die Anregungen zu dieser Arbeit und für viele wertvolle Diskussionen.

Den Herren O. G. Folberth, R. Gremmelmaier, R. Grimm und O. Madelung danke ich für die Herstellung der Präparate sowie Herrn E. Weißhaar für die Hilfe bei den Wechselstrommessungen. Herrn Pfister danke ich für Röntgenuntersuchungen.

${ }^{9}$ G. L. Pearson u. M. Tanenbaum, Physic. Rev. 90, 153 [1953]. 